\title{
fabricación industrial de arcilla expandida
}

\author{
IJ. C. CUBAUD Y M. MURAT \\ Silicates Industriels, n. ${ }^{\circ}$, mayo_1968, págs. 145-152
}

\section{introducción}

La construcción da cada vez más importancia a los hormigones preparados con áridos ligeros. De hecho, estos materiales permiten, suponiéndoles unas resistencias mecánicas suficientes, sustituir los elementos del hormigón pesado por otros que, a igualdad de espesor, proporcionan un aislamiento térmico muy superior. Ciertos áridos ligeros se encuentran en estado natural (piedra pómez) o son subproductos industriales (escorias). Pero cada día se fabrica una proporción mayor de materiales ligeros industrialmente, entre ellos: la arcilla expandida.

La expansión, obtenida a alta temperatura $\left(1.100^{\circ}-1.200^{\circ} \mathrm{C}\right)$, da al gránulo de arcilla una estructura esponjosa, que conserva en su enfriamiento, y tiene una resistencia mecánica suficiente.

En este trabajo se estudia el mecanismo de la expansión y la fabricación industrial de la arcilla expandida. Posteriormente se expondrán las propiedades de este material y su aplicación en la construcción.

\section{GENERALIDADES}

El descubrimiento de la arcilla expandida se remonta al año 1885. La primera explotación en escala industrial surge en 1918, según una patente del americano S. J. Hayde (1), fabricándose de manera racional y empleándose como árido del hormigón ligero.

Según Everhart (12), en 1966 existían en Estados Unidos 60 fábricas con una producción anual de $4.000 .000 \mathrm{~m}^{3}$ de arcilla expandida. Rusia y Europa Occidental poseen, respectivamente, 30 y 20 centros de fabricación. El consumo anual es de $700.000 \mathrm{~m}^{3}$ en Dinamarca, $400.000 \mathrm{~m}^{3}$ en Alemania y $300.000 \mathrm{~m}^{3}$ en Suiza.

Entre los diversos procedimientos de expansión de arcillas, el LECA, siglas de "Light Expanded Clay Aggregate", patentado hace veinte años en Dinamarca, es el único empleado en Francia en la fábrica de Watten (Paso de Calais). La producción, comenzada en 1963 , es de unos $200.000 \mathrm{~m}^{3}$ por año. 


\section{LA ARCILLA Y LA EXPANSION}

La expansión se debe esencialmente a la formación de gas, que no puede escapar de la envoltura exterior aglomerada por vitrificación en el curso de un tratamiento térmico. Este gas, crea, pues, un aumento de volumen de la arcilla piroplástica.

La eleccićn de la arcilla y la naturaleza y origen del hinchamiento dependen respectivamente de los dos factores siguientes: composición química y composición mineralógica. Veamos cada factor por separado:

\section{1. Elección de la arcilla - Composición química}

La necesidad de crear una fase fundida de viscosidad suficientemente elevada para encerrar un gas, entraña ciertas restricciones en la elección de la arciila. En efecto, los contenidos en sílice, alúmina y fundentes (cal, magnesia, óxidos de hierro, álcalis) no deben sobrepasar ciertos límites, ya que, de lo contrario, la arcilla no fundiría a una temperatura suficientemente baja o fundiría en una masa insuficientemente viscosa (13) y (2). Tomando como base las investigaciones de Conley y colaboradores (11) y de Riley (4) se ha podido determinar, en un diagrama triangular, la zona de composición química que responde a las condiciones de viscosidad y de hinchamiento (fig. 1). Las arcillas situadas fuera de esta zona no tienen aptitud alguna para la expansión. Por otro lado, ciertos puntos en el interior de la zona definida precedentemente corresponden a productos que pueden formar una masa con la viscosidad necesaria, pero que no tienen la posibilidad de producir desprendimientos gaseosos en el curso de su tratamiento térmico, lo cual se relaciona con su composición mineralógica.

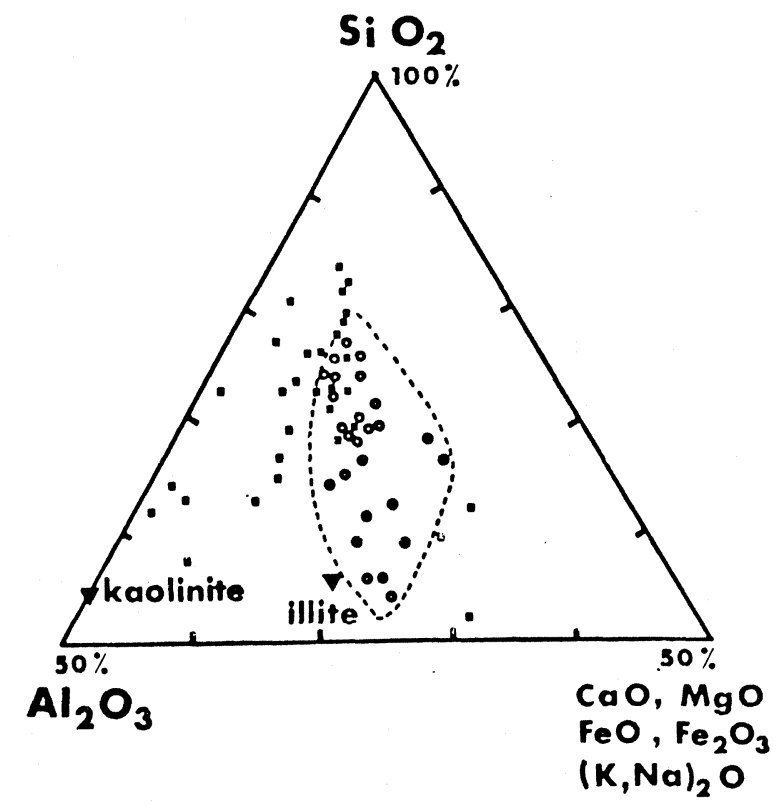

Fig. 1.-Diagrama que demuestra las composiciones requeridas para la expansión de la arcilla.

Arcillas adecuadas para la expansión.

arcillas no favorables para la expansión. $\nabla$ Minerales arcillosos.

Ei grado de vitrificación, también es función de la composición química: la obtención de gránulos ligeros de buena calidad implica que la envoltura externa de cada gránulo sea impermeable y resistente. La zona de temperaturas donde la vitrificación debe intervenir se sitúa entre el punto donde comienza la fusión y el punto en que la visco- 
sidad es muy baja para que la expansión tenga lugar. Las arcillas que contienen sodio y potasio producen mezclas muy viscosas. Las que contienen magnesio y calcio conducen a mezclas de baja viscosidad. El óxido de hierro se considera como un fundente intermedio. Mathews (14) ha establecido una relación entre la aptitud a la vitrificación y las proporciones de $\mathrm{K}_{2} \mathrm{O}+\mathrm{Na}_{2} \mathrm{O}$ y $\mathrm{CaO}+\mathrm{MgO}$, obteniéndose una vitrificación correcta cuando:

$$
\frac{\mathrm{K}_{2} \mathrm{O}+\mathrm{Na}_{2} \mathrm{O}}{\mathrm{CaO}+\mathrm{MgO}}>1
$$

\section{2. Naturaleza y origen del hinchamiento de las arcillas. Influencia de la composición mineralógica (2) a (13)}

Numerosos trabajos establecen el mecanismo de la expansión y la naturaleza y origen del hinchamiento de las arcillas. Everhart (15), con ayuda del análisis térmico diferencial, ha estudiado la importancia de la composición mineralógica sobre la aptitud a la expansión. Las arcillas que ha empleado estaban constituidas esencialmente de illita y de caolinita, cuyas fórmulas generales (24) son respectivamente $\left(\mathrm{Si}_{i_{4-\mathrm{x}}} \mathrm{Al}_{\mathrm{x}}\right)\left(\mathrm{Al}_{2}\right) \mathrm{O}_{10}(\mathrm{OH})_{2}(\mathrm{~K})_{\mathrm{x}}$ para la illita y $\left(\mathrm{Si}_{2}\right)\left(\mathrm{Al}_{2}\right) \mathrm{O}_{5}(\mathrm{OH})_{4}$ para la media malla de la caolinita. La relación illita/caolinita es igual a 2,7 para los productos que expansionan y se convierte en 1,6 para los que no expansionan.

A fin de determinar los minerales responsables de la expansión, Riley (4) ha preparado mezclas artificiales y ha llegado a la conclusión de que la pirita, la hematites, la dolomita y la calcita pueden producir un gas a una temperatura suficientemente elevada para que se manifieste la expansión. Los estudios de Austin, Nunes y Sullivan (16) han permitido demostrar que la expansión de las arcillas se produce incluso en atmósfera de gas inerte (nitrógeno); por lo tanto, este fenómeno tiene su origen en los propios constituyentes de la arcilla y no es el resultado de una acción química entre la arcilla y la atmósfera del horno. Estos autores han establecido más tarde, que los gases formados son anhídrido carbónico $\left(\mathrm{CO}_{2}\right)$, anhídrido sulfúrico $\left(\mathrm{SO}_{3}\right)$ y vapor de agua $\left(\mathrm{H}_{2} \mathrm{O}\right)$. Ehlers (25) en particular ha aplicado la cromatografía al análisis de los gases que provocan la expansión de una serie de arcillas específicas: ha demostrado que, en esta investigación, el fenómeno de expansión está producido por la formación de $\mathrm{CO}_{2}$ que resulta de la descomposición térmica de la calcita $\mathrm{CO}_{3} \mathrm{Ca}$ y de la ankerita $\mathrm{Ca}(\mathrm{Fe}, \mathrm{Mg})\left(\mathrm{CO}_{3}\right)_{2}$. Esta descomposición se produce en el interior del nódulo cuya superficie se vitrifica por un tratamiento térmico rápido. Stamboliev (27), ha mostrado la influencia de la formación de $\mathrm{SO}_{2}$ y de $\mathrm{CO}$ en la expansión de arcillas ricas en carbono orgánico, álcalis y sulfatos.

A partir de los datos suministrados por la literatura se ha hecho un balance de las diferentes reacciones de descomposición de los constituyentes mineralógicos que conducen a un desprendimiento gaseoso:

1. El carbono libre, presente en la arcilla, reduce el óxido de hierro en $\mathrm{FeO}$ y $\mathrm{CO}_{2}$ :

$$
2 \mathrm{Fe}_{2} \mathrm{O}_{3}+\mathrm{C} \longrightarrow 4 \mathrm{FeO}+\mathrm{CO}_{2}^{\nearrow}
$$

Igualmente puede formarse monóxido de carbono, según la reacción:

$$
\mathrm{Fe}_{2} \mathrm{O}_{3}+\mathrm{C} \longrightarrow 2 \mathrm{FeO}+\mathrm{CO}
$$


2. La pirita: ésta se disocia, en primer lugar, entre $595^{\circ}$ y $1.000^{\circ} \mathrm{C}$, dando azufre y pirrotina, según la reacción:

$$
\mathrm{FeS}_{2} \longrightarrow \mathrm{FeS}+\mathrm{S} \text { (vapor) }
$$

Más tarde, la pirrotina y el azufre se oxidan a más alta temperatura con desprendimiento de anhídrido sulfuroso (20) y (21):

$$
\begin{aligned}
2 \mathrm{FeS}+3 \mathrm{O}_{2} \longrightarrow & 2 \mathrm{FeO}+2 \mathrm{SO}_{2} \\
\mathrm{~S}+\mathrm{O}_{2} \longrightarrow & \mathrm{SO}_{2} .
\end{aligned}
$$

Inestable en presencia de sílice, el $\mathrm{FeO}$ se transforma en silicato de hierro. El azufre puede provenir igualmente de la reducción de $\mathrm{Fe}_{2} \mathrm{O}_{3}$ por la pirita (26):

$$
\mathrm{Fe}_{2} \mathrm{O}_{3}+\mathrm{FeS}_{2} \longrightarrow 3 \mathrm{FeO}+2 \mathrm{~S}^{\prime} \text { (vapor). }
$$

3. Hematites y limonita: a partir de $1.000^{\circ} \mathrm{C}$ estos minerales se transforman en óxidos del tipo $\mathrm{Fe}_{3} \mathrm{O}_{4}$ con eliminación de oxígeno gaseoso, responsable de la expansión:

$$
6 \mathrm{Fe}_{2} \mathrm{O}_{3} \rightleftharpoons 4 \mathrm{Fe}_{3} \mathrm{O}_{4}+\mathrm{O}_{2} \text {. }
$$

Aunque Orton y Staley (19) hayan desechado esta hipótesis, pues consideran que el sulfuro de hierro es el verdadero responsable del fenómeno de la expansión, numerosos autores han confirmado este proceso (17) y (18). Esta descomposición se facilita por la presencia de óxidos tales como el $\mathrm{MgO}$, procedente de la descomposición de la dolomía (26) y (27).

4. Dolomía y calcita: la descomposición térmica de la dolomía, $\mathrm{CaMg}\left(\mathrm{CO}_{3}\right)_{2}$, y de la calcita, $\mathrm{CaCO}_{3}$, conduce a un desprendimiento de anhídrido carbónico, $\mathrm{CO}_{2}$ : en el caso de la dolomía se piensa que la gran dilatación observada en la arcilla no puede explicarse más que por una descomposición en dos etapas, a $750^{\circ} \mathrm{C}$ y a $890^{\circ} \mathrm{C}$. Estas temperaturas son muy bajas y también Riley (4) ha supuesto que se formaría un compuesto intermedio que retiene parte de $\mathrm{CO}_{2}$ y no se disocia más que a temperatura más elevada. Se ha demostrado que la dolomía es un excelente agente de expansión (12), mejor que la calcita. La presencia de ankerita, $\mathrm{Ca}(\mathrm{Fe}, \mathrm{Mg})\left(\mathrm{CO}_{3}\right)_{3}$, puede igualmente conducir a un desprendimiento de $\mathrm{CO}_{2}(25)$.

5. Hornablenda y biotita: estos minerales se disocian durante el hinchamiento de las arcillas, provocando un desprendimiento de vapor de agua. La reacción, para la biotita por ejemplo, es la siguiente:

$$
\mathrm{K}_{2}(\mathrm{Mg}, \mathrm{Fe})_{6}\left(\mathrm{Al}, \mathrm{Si}_{3} \mathrm{O}_{10}\right)_{2}(\mathrm{OH})_{4} \longrightarrow \mathrm{K}_{2} \mathrm{O}, 6(\mathrm{Mg}, \mathrm{Fe}) \mathrm{O}, \mathrm{Al}_{2} \mathrm{O}_{3}, 6 \mathrm{SiO}_{2}+2 \mathrm{H}_{2} \mathrm{O}
$$

Esta agua puede actuar sobre el $\mathrm{FeO}$ procedente de la oxidación de la pirrotita, por ejemplo, pudiéndose observar entonces un desprendimiento de hidrógeno:

$$
2 \mathrm{FeO}+\mathrm{H}_{2} \mathrm{O} \longrightarrow \mathrm{Fe}_{2} \mathrm{O}_{3}+\mathrm{H}_{2}^{\nearrow}
$$

6. Los sulfatos: los sulfatos de calcio, magnesio u otros sulfatos intermedios for- 
mados en el curso del tratamiento térmico, se descomponen liberando $\mathrm{SO}_{2}$ y $\mathrm{SO}_{3}$, según reacciones del tipo:

$$
\begin{gathered}
\mathrm{CaSO}_{4} \longrightarrow \mathrm{CaO}+\mathrm{SO}_{2} \\
2 \mathrm{CaSO}_{4} \longrightarrow 2 \mathrm{CaO}+2 \mathrm{SO}_{2}^{\prime}+\mathrm{O}_{2} \\
2 \mathrm{Fe}_{2}\left(\mathrm{SO}_{4}\right)_{3} \longrightarrow 2 \mathrm{Fe}_{2} \mathrm{O}_{3}+6 \mathrm{SO}_{2}^{\prime}+3 \mathrm{O}_{2}^{\prime} .
\end{gathered}
$$

Las opiniones en cuanto a las causas de la expansión son a veces muy diversas y así, por ejemplo, Johnson (12) ha encontrado que la hematites y el yeso no conducen a la expansión.

Conley y colaboradores (11) dan la siguiente tabla relativa a la cantidad mínima de materiales que producen $100 \mathrm{~cm}^{3}$ de gas a $1.260^{\circ} \mathrm{C}$, para expansionar 10 gr de muestra.

TABLA 1

\begin{tabular}{|l|l|l|}
\hline Constituyente & Peso (\%) & Gas formado \\
\hline $\mathrm{FeS}$ & 0,700 & $\mathrm{~S}$ vap. o $\mathrm{SO}_{2}$ \\
$\mathrm{FeSO}_{4}$ & 1,208 & $\mathrm{SO}_{3}$ \\
$\mathrm{FeCl}_{2}$ & 1,000 & $\mathrm{Cl}_{2}$ \\
$\mathrm{NaCl}$ & 0,930 & $\mathrm{Cl}_{2}$ \\
$\mathrm{CaSO}_{4}$ & 1,082 & $\mathrm{SO}_{3}$ \\
$\mathrm{Na}_{2} \mathrm{CO}_{3}$ & 0,843 & $\mathrm{CO}_{2}$ \\
$\mathrm{CaCO}_{3}$ & 0,796 & $\mathrm{CO}_{2}$ \\
$\mathrm{CaMg}_{\left(\mathrm{CO}_{3}\right)_{2}}$ & 0,855 & $\mathrm{CO}_{2}$ \\
$\mathrm{Fe}_{2} \mathrm{O}_{3}$ & 7,632 & $\mathrm{O}_{2}$ \\
\hline
\end{tabular}

Se ve pues que, otros compuestos tales como los cloruros, son susceptibles de provocar un desprendimiento gaseoso favorable a la expansión.

\section{3. Adición de agentes que favorecen la expansión}

Si la arcilla de partida no contiene ni pirita, hematites, dolomita o calcita, se puede pensar que una adición de estos compuestos mejorará la fabricación de los granulados ligeros. Esto es lo que han realizado Everhart y colaboradores (12), que han estudiado sistemáticamente la influencia de las siguientes adiciones sobre una arcilla de Ohio:

$$
\begin{aligned}
& \text { - Calcita } \\
& \text { - "Nefelina sienita" } \\
& \text { - Yeso } \\
& \text { - Hematites } \\
& \text { - Pirita } \\
& \text { - Sosa comercial. }
\end{aligned}
$$


En cada caso han considerado varias fracciones granulométricas bien definidas y han hecho variar el porcentaje de adición. Las muestras se han sometido a tratamientos térmicos entre $1.150^{\circ}$ y $1.320^{\circ} \mathrm{C}$ durante 15 minutos. Las curvas que representan la densidad del gránulo en función de la temperatura de cocción han permitido deducir las siguientes conclusiones:

- los productos de adición que actúan a la vez como produciores de gas y como fundente son más interesantes que los que poseen sólo una de estas propiedades;

- con agentes muy favorables a la expansión, la densidad aparente decrece con la elevación de la temperatura de cocción;

- la densidad aparente decrece generalmente cuando aumenta la proporción de aditivo ;

- la densidad aparente disminuye cuando se hace disminuir el tamaño de partícula del producto adicionado.

Finalmente, los productos que permiten mejor expansión son, esencialmente, la pirita, la calcita y la "nefelita sienita", aunque esta última no posea las dos cualidades de fundente y generador de gas.

\section{FABRICACION INDUSTRIAL DE LOS GRANULADOS DE ARCILLA EXPANDIDA}

Las fábricas se sitúan generalmente en la proximidad de las canteras. No se detallarán en este trabajo la explotación de los yacimientos, la extracción o el transporte de materias primas, sino que sólo se describirán los distintos métodos de fabricación.

El granulado de arcilla expandida debe tener una resistencia propia muy elevada, compatible con un peso lo más reducido posible. Estas condiciones pueden cumplirse mejor por un gránulo de forma esférica y una superficie externa cerrada, no porosa y de preferencia vitrificada. Por el contrario, el interior deberá presentar una gran porosidad para conseguir el mínimo peso. No obstante, la forma esférica no es general y se fabrican gránulos de forma diferente.

A los imperativos físicos que se acaban de mencionar deben añadirse los imperativos económicos siguientes:

- pequeños gastos de inversión y funcionamiento de la instalación de producción;

- sistema de horno simple con instalación de regulación y control automático, adaptando la capacidad de producción a las posibilidades de almacenamiento;

- empleo de arcilla apta para la expansión, incluso sin aditivos.

La necesidad de obtener, en el caso general, gránulos completamente esféricos, supone que el horno esté alimentado con gránulos previamente dispuestos a su dimensión con un dispositivo adecuado de tamizado. A fin de evitar todo riesgo de aglomeración del material, y para obtener una densidad de grano uniforme, es necesario que durante el caientamiento se remuevan los materiales en el horno. Además, la exigencia primordial para explotar al máximo las posibilidades de expansión de la arcilla y obtener una corteza externa del grano con elevada resistencia, es el empleo de un tratamiento térmico muy rápido. 
En el caso en que las arcillas empleadas manifiesten una expansión insuficiente, se mejorará su comportamiento con el aporte de agentes de hinchamiento tales como el sulfato de sosa, los hidrocarburos pesados (aceites diesel en particular), el coque, los sulfatos, el silicato sódico, los carbonatos alcalinos, la calcita o la hematites (11) y (12). Con las arcillas mediocres puede también hacerse una calcinación previa antes del moldeo, pero es necesario que las arcillas se reblandezcan entre $1.050^{\circ}$ y $1.250^{\circ} \mathrm{C}(32)$.

La expansión de la arcilla puede realizarse de diferentes maneras:

- por paso del material convenientemente preparado en gránulos, a través de un horno rotatorio a temperatura suficientemente elevada $\left(1.100^{\circ}-1.200^{\circ} \mathrm{C}\right)$;

- introduciendo el material en un horno vertical y fluidizándolo por una corriente gaseosa ascendente;

- pasando la arcilla sobre una parrilla móvil, donde una temperatura de unos $1.200^{\circ} \mathrm{C}$ provoca la expansión.

A continuación se insistirá de un modo particular en los dos primeros procedimientos, siendo el tercero menos frecuente, aunque se utiliza sobre todo en Estados Unidos (29) y en la República de Bielo-Rusia (34).

\section{1. Horno rotativo}

Russenmeyer (22) ha descrito una instalación piloto que permite realizar múltiples ensayos con el fin de determinar las condiciones óptimas en función de diferentes materias primas. El compartimiento de secado y precalentamiento, constituido por un intercambiador de calor de parrilla móvil, es regulable entre amplios límites en velocidad, altura de la capa de material y repartición de la temperatura de los gases. El horno rotatorio, situado a continuación de este compartimiento, puede regularse en longitud, en pendiente y en velocidad de rotación, pudiéndose variar pues los parámetros en función de las diferentes primeras materias. El quemador, que funciona con aceite ligero, permite regular la temperatura de la llama y la atmósfera del horno en un amplio margen. Estando provista la instalación de un gran número de puntos de medida (temperatura, composición de la mezcla gaseosa, presión y consumo de combustible) es posible efectuar balances térmicos completos.

Además de la homogeneidad en la cocción, existen otros factores que intervienen en la calidad del producto final. En el caso del procedimiento por vía seca, estos factores son: el tipo de fragmentación, la finura después de la molienda, la homogeneización, la granulación, el tamizado, el secado, el proceso de enfriamiento y el método de cocción. Todos ellos deben permitir regular a voluntad el grado de expansión y, por lo tanto, la dureza del producto final.

En general, existen dos procedimientos en servicio: vía seca y vía húmeda.

En el procedimiento por vía seca (Fig. 2), la materia prima (arcilla con $20 \%$ de humedad) se seca hasta un grado de humedad de $8-10 \%$; se muele, se precalienta, se granula por tamizado y se envía a un horno rotatorio donde el choque térmico provoca la expansión y la vitrificación de la superficie de los nódulos. El material pasa entonces a un tambor de enfriamiento antes de triturarse y almacenarse. El producto final tiene forma esférica. 
En el procedimiento por vía húmeda (Fig. 3), la granulación se realiza con un gasto de energía relativamente pequeño por medio de una prensa de hacer pastillas. Las pastillas de forma cilíndrica, se redondean posteriormente por frotamiento en los tambores de granulación, transformándose en partículas esféricas.

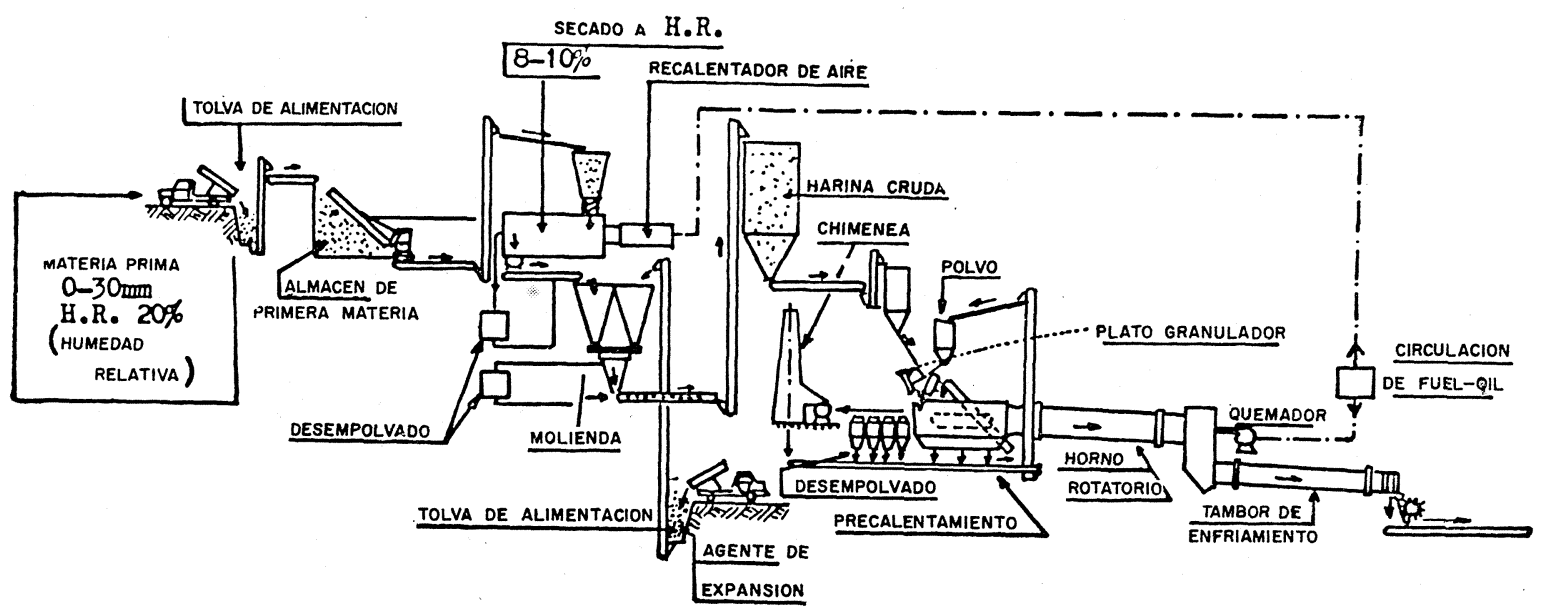

Fig. 2.-Procedimiento por vía seca.

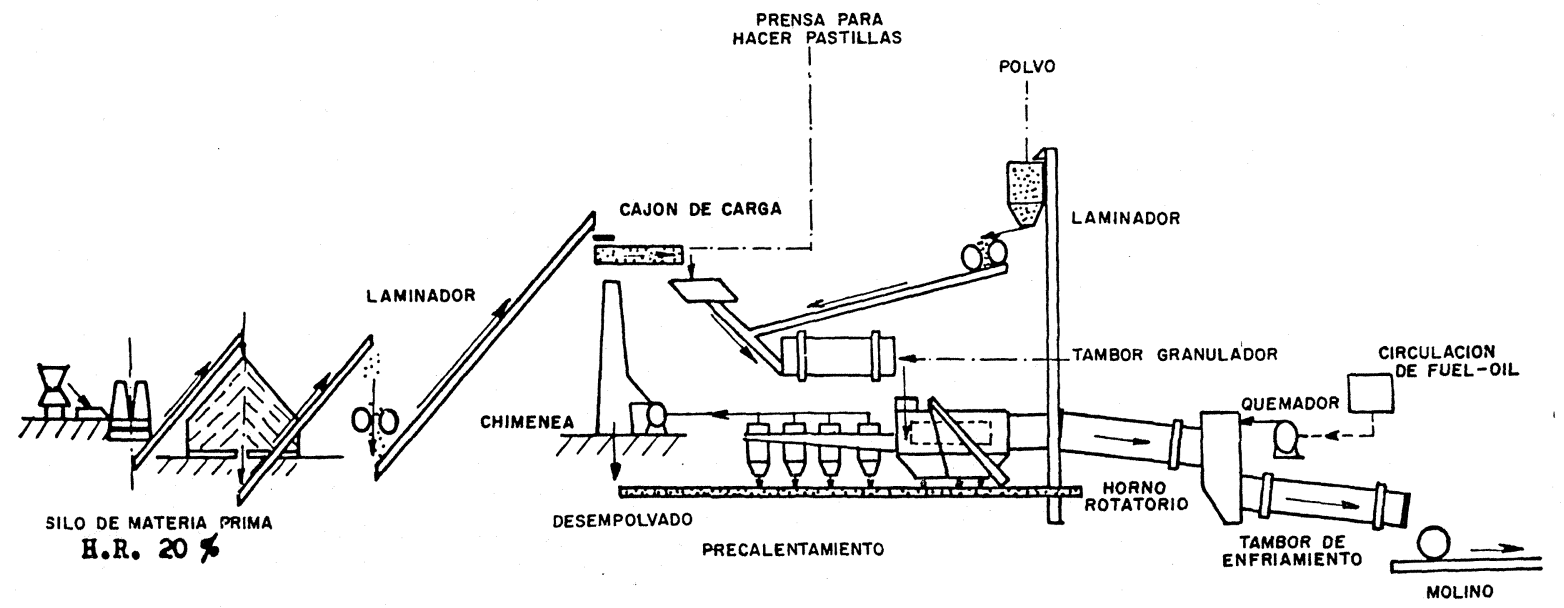

Fig. 3.-Procedimiento por vía húmeda.

\section{Procedimiento "LECA" (Light Expanded Clay Aggregate)}

Después de un amasado y una molienda prolongados, la arcilla se introduce húmeda (contenido de agua: $25 \%$ ) en forma de finas láminas en un horno rotatorio de fuego continuo y de una longitud de $65 \mathrm{~m}$, constituido de dos elementos de diámetro distinto y dotados de velocidad variable. La primera parte del horno (2,20 m de diámetro) sirve para el secado. Las temperaturas son $200^{\circ} \mathrm{C}$ a la entrada y $800^{\circ} \mathrm{C}$ a la salida. Aquí la arcilla se deshidrata y toma su forma definitiva, en marcha hacia la segunda parte del horno cuyo 
diámetro es igual a 3,5 m. En esta fase, el gránulo desarrolla su expansión y su envoltura se vitrifica, siendo la temperatura del horno muy próxima a los $1.100^{\circ} \mathrm{C}$.

La arcilla expandida sale por el extremo del horno en forma de nódulos ovoidales.

Este procedimiento se puso a punto en Dinamarca hace unos 20 años. El material obtenido tiene un comportamiento extraordinario y pronto se conocieron sus múltiples aplicaciones justificadas por sus propiedades aislantes. En seguida se extendió en Europa (Alemania, Suiza, Austria, Inglaterra) y en Estados Unidos. En Francia sólo existe un yacimiento de arcilla que permite la fabricación de gránulos del tipo LECA. Se encuentra en Watten (Paso de Calais) y se ha instalado una fábrica a pie de cantera. La arcilla se trata sin maduración previa. La fábrica produce unos $200.000 \mathrm{~m}^{3}$ /año de arcilla expandida (30).

\section{2. Procedimiento del lecho fluidificado}

Este procedimiento patentado, descrito por Denner (1) y (23), lleva consigo las siguientes fases:

La arcilla procedente de cantera sufre una molienda previa antes de ser almacenada en un silo. A partir del silo, la arcilla todavía húmeda se dirige a un molino donde se pulveriza finamente y se homogeneiza. Inmediatamente se somete a un tratamiento de granulación, que se realiza por inyección de 2 a $4 \%$ de agua y de un aditivc convenientemente elegido.

Los gránulos (de 1 a $10 \mathrm{~mm}$ de diámetro) pasan a continuación por un tambor de secado horizontal (Fig. 4), de unos $10 \mathrm{~m}$ de longitud y $1,5 \mathrm{~m}$ de diámetro. El funcionamiento es en contra-corriente para evitar que el producto, que contiene alrededor de $18 \%$ de humedad, se enfrente directamente con las partes calientes $\left(600^{\circ} \mathrm{C}\right)$ del tambor.

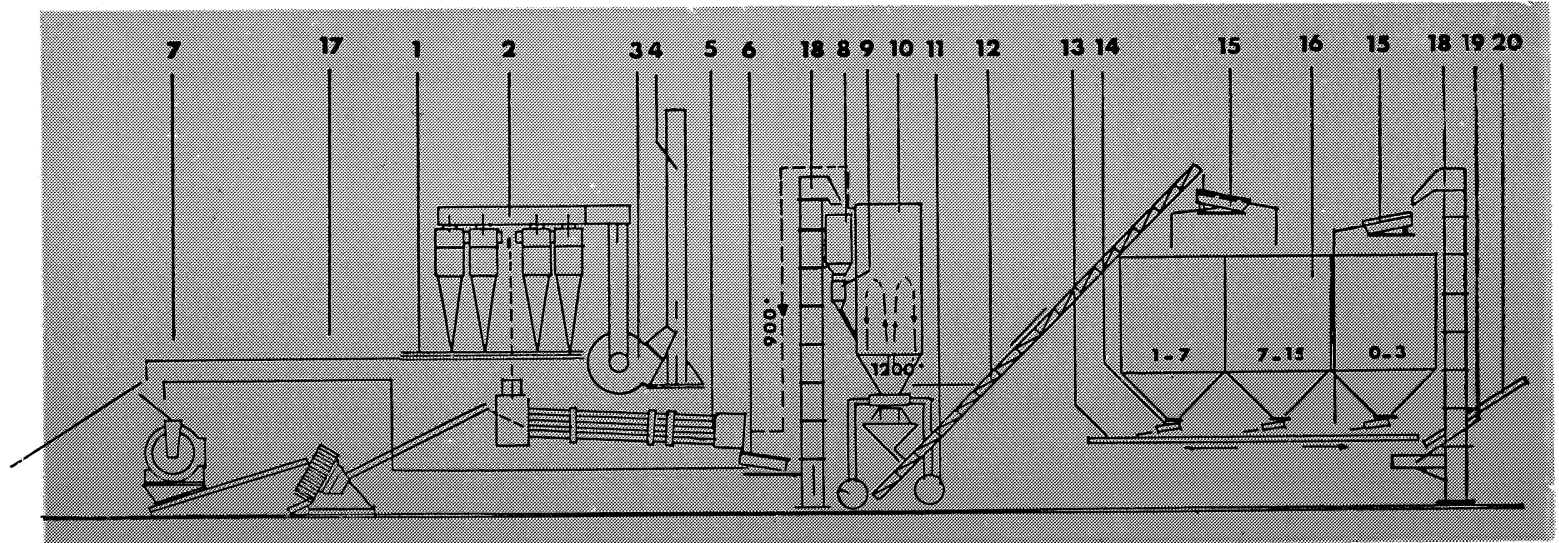

Fig. 4.-Esquema del proceso del lecho fluidificado.

1.- Rosca transportadora.

2.-Batería de ciclones.

3.-Ventilador.

4.-Chimenea.

5.-Tambor de secado.

6.-Tamiz.

7.-Molino.

8.-Tolva.

9.-Válvula.

10.-Horno.
11.-Soplado.

12. y 13.-Cintas transportadoras.

14.-Distribuidor.

15.-Tamiz.

16.-Silos.

17.-Plato granulador.

18.-Elevador.

19.-Molino.

20.-Cinta transportadora. 
A la salida del tambor, y después de tamizar los granos, se envían a una tolva que vierte periódicamente una cantidad determinada de material en el horno del lecho fluidificado. Un chorro de gas que entra en la base de la cámara de combustión recoge el material y le levanta. El aire necesario para la combustión se mezcla con los hidrocarburos en un quemador; los gases de combustión resultantes $\left(1.200^{\circ} \mathrm{C}\right)$ abandonan el horno a $900^{\circ} \mathrm{C}$ y se emplean para calentar el tambor de secado en el cual pasan a $600^{\circ} \mathrm{C}$, después de incorporarles aire frío. Los gases residuales a la salida del tambor tienen unos $110^{\circ} \mathrm{C}$; sufren un desempolvamiento en un aparato de tipo ciclón y los polvos recogidos se reciclan hacia el molino. La duración total de la estancia de la arcilla en el horno es de 60 segundos.

Al final de la combustión, el material se expulsa aí exterior del horno a.briendo una válvula. Más tarde se almacena en los silos.

Al contrario que los hornos rotativos, el horno de lecho fluidificado puede ponerse en marcha o pararse en cada momento, sin peligro alguno para el horno o su revestimiento interno. Puede ponerse en marcha después de varias horas de enfriamiento. La puesta en marcha de una instalación totalmente parada necesita menos de una hora. Tal fábrica puede, gracias a una amplia automatización, producir 200 t/día de gránulos de arcilla con un efectivo de cuatro hombres. El horno vertical tiene las sisuientes dimensiones: diámetro, $2,5 \mathrm{~m}$; altura, 20 metros.

En Estados Unidos, se encuentran en funcionamiento algunos procedimientos derivados del lecho fluidificado (31), sobre todo instalaciones que funcionan de modo continuo y en las que se funciona de la siguiente manera:

La arcilla finamente molida y con un grado de humedad del $28 \%$, se pasa a través de una placa perforada que gira a 3.000 r.p.m. con perforaciones de $0,7 \mathrm{~mm}$. El estiramiento produce nódulos que al caer se secan por un flujo de aire ascendente. El material pasa a continuación a la parte alta del horno calentado con gas, a través de un alimentador de lecho fluidificado que realiza un precalentamiento. Entonces pasa a la cámara de cocción, a través de tubos calefactores que regulan la producción. La temperatura de la zona de cocción es del orden de $1.540^{\circ}-1.650^{\circ} \mathrm{C}$. El horno especial costó unos 300.000 dólares U.S.A. en 1964 .

\section{3. Horno de parrilla móvil}

Este sistema (sistema Dwigt-Lloyd) fue inventado a principio de siglo aglomerando por vidriado minerales metálicos pulverulentos (2). Se adapta bien al tratamiento de arcillas diversas mezcladas con coque pulverizado. La mezcla previamente molida se coloca sobre una parrilla móvil en una capa que se calienta por la superficie. El fuego se propaga a través de todo el espesor por efecto de una aspiración de aire. El calentamiento realizado en atmósfera reductora, es suficiente para provocar un principio de fusión y un hinchamiento simultáneo de la carga.

Este procedimiento parece estar llamado a un porvenir más brillante que el del horno rotatorio. Conley y colaboradores (37) han demostrado que ciertos materiales que presentan las características necesarias para obtener áridos ligeros, no han dado los resultados que se esperaba en el horno rotatorio, pues alcanzan su punto de fusión antes de que se manifieste el hinchamiento: se producen apelmazamientos en las paredes del horno, 
que es preciso evitar. La gran superficie de radiación de los hornos rotatorios y la falta de aislamiento suficiente reducen su rendimiento térmico. Por eso, debe reservarse la expansión en hornos rotatorios a arcillas de gran calidad (38).

El horno de parrilla móvil presenta otras ventajas, en particular: la rapidez de encendido, de vitrificación y de hinchamiento (39), la ausencia de revestimiento refractario, la flexibilidad de funcionamiento, un costo de producción más reducido que en el horno rotatorio (38), etc.

Este procedimiento se emplea en particular en Estados Unidos, para la fabricación de "Masslite" en Plainville (Massachusset), de "Shalite" en Knoxville (Tennessy), etc. (29).

En la literatura se encuentran varias descripciones, modificaciones o particularidades, según la naturaleza de la arcilla empleada o el país en el cual se realiza la fabricación.

Se pueden preparar, por ejemplo, materiales ligeros por expansión de ciertas mezclas a base de cenizas volantes y barbotina de arcilla o bentonita (33). Tendría también la ventaja de realizar una fabricación mixta: productos para ladrillería y granulados ligeros en una fábrica de arcilla cocida, pudiendo emplearse como materias primas las arcillas húmedas, los esquistos blandos, etc. (35).

Mencionemos la fabricación del hormigón "Céramsite". El granulado es una gravilla cuya fabricación consiste en hilar una arcilla común en Rusia, después de haberla mezclado con agua. Estos filamentos se cortan a continuación y su expansión conduce a granos que constituyen un material cerámico muy ligero (36).

La mayor parte de las fabricaciones se realizan, sin embargo, con la ayuda de tres métodos fundamentales: por lecho fluidificado, en horno rotatorio o por cocción en parrilla móvil.

No obstante, no debe olvidarse que un árido para hormigón debe tener un precio bajo en el mercado. El costo de transporte debe reducirse al mínimo, si se quiere conservar la noción de material barato, lo que obliga a instalar las fábricas en las proximidades de los centros de consumo.

\section{CONCLUSION}

Se ha descrito el fenómeno de la expansión y los principales procedimientos de fabricación industrial de los áridos ligeros a base de arcilla. Las indudables ventajas de estos materiales (poco peso específico, propiedades de aislamiento térmico, resistencias mecánicas teniendo en cuenta el ahorro de peso conseguido, etc.) permiten su empleo en las siguientes fabricaciones:

-- hormigón cavernoso, no resistente, buen aislante térmico;

- hormigón aislante, y resistente a la vez;

- hormigón armado y estructuras mixtas acero-hormigón.

El posible porvenir de este material, unido a los imperativos económicos antes citados, llevarán consigo en los próximos años numerosas investigaciones a este respecto. 


\section{BIBLIOGRAFIA}

(1) H. V. DenNeRt : Ziegelindustrie, Dtsch, 1966, 19, 22, 827-33.

(2) A. G. L'Industrie Céramique, 1952, octubre, 312-314.

(3) T. Whitacker: National Building Studies Spec. Rep. núm. 12, 1953 -Londres.

(4) Ch. M. RIley: J. Amer. Ceram. Soc. 34, 1951, núm. 4, págs. 121-128.

(5) R. E. Davies y J. W. Kelly: Lightweight Symposium on Mineral aggregates 1948. Amer. Soc. Materials Spec. Techn. Publication núm. 83, págs. 160-171.

(6) C. R. Austin, J. L. Nunes y J. D. Sullivan: Amer. nst. of Moning and Metallurgical Engineers 1942, Tech. Publ. núm. 1.486.

(7) F. KAEMPFE: Zement Kalk Gips, 11, 1958, núm. 10.

(8) H. Fastabend, J. L. Ruyter: Tonindustrie-Zeitung 83, 1959, núm. 22.

(9) W. Schellmann, H. Fastabend: Ziegelindustrie 1963, núm. 24.

(10) K. H. LUCKE: Silikatechnik 1960, núm. 7.

(11) J. E. Conley, H. Wilson, J. A. KLINEFECTER: U.S. Bureau of Mines Reports 1948 Investigation número 4401 , pág. 121.

(12) J. O. Everhart, E. G. Ehlers, J. E. Johnson, J. H. Richardson: Engineering Experiment Station 1958, Bulletin núm. 169.

(13) W. S. Ramachandran, N. C. Majumdar, N. K. Patwardhan: Indian Concrete Journal, 1961, diciembre, $453-456$.

(14) J. G. Mathews: Canada Memo-Series, núm. 120, 1952.

(15) J. O. Everhart: Brick and Clay Record, 1959, mayo, págs. 58-59.

(16) C. R. Austin, J. L. Nunes, J. D. Sullivan: Trans. Am. Inst. Moning and Met. Eng. 1942, núm. 148, págs. 149-159.

(17) T. E. JACKson: Trans. Ceram. Soc. 1903, págs. 37-43.

(18) J. C. Hostetter, H. S. Roberts: Jour. Am. Ceram. Soc. 1921, noviembre, págs. 927-938.

(19) E. Orton, H. F. Staley: Third Report, National Brick Manufacturers Association T. H. Randall \& Co Indianapolis - Indiana 1903.

(20) W. Hempel, C. Schubert: Z. Elektrochem. 1912, vol. 18, págs. 729-733.

(21) E. T. Allen, R. H. Lombard: Am. Jour. Sci. 1917, vol. 43, págs. 175-195.

(22) H. RusSEMeYer: Aufbereitungs-Technik 1966, núm. 4, págs. 220-223.

(23) H. V. Dennert: Aufbereitungs-Technik 1966, núm. 6, págs. 314-317.

(24) S. Caillere y S. Henin: Minéralogie des Argiles, Masson et Cie. Ed. París 1963, págs. 168 y 211.

(25) E. G. EHLers: Bull. Amer. Ceram. Soc. 1958, 37, 95-99.

(26) R. D. Hill y D. N. Crook: Austr. J. Appl. Sci. 1960, 39, 8, 408-12.

(27) D. Woodhouse y J. White: Trans. Brit. Ceram. Soc. 1955, 54, 333.

(28) H. T. Stamboliev: Amer. Ceram. Soc. 1961, 40, 4, 257.

(29) J. Portmann: Betonstein Ztg. Dtsch. 1966, 2, 93-100.

(30) P. L. D. Bâtir, Fr., (junio 1966), XLVII.

(31) S.C.R. Veri-lite fait espérer des progrès dans les produits argileux. Bull. Amer. Ceram. Soc. (mayo 1964), 43, 5, 408-409.

(32) G. A. Kirkendale: Bull. Ceram. Res. Ass. Israël (abril 1960), 10, 19-22.

(33) N. MoHLeR: Brick Clay Rec. (septiembre 1963), 143, 3, 76-77.

(34) W. J. Kunaschrewitsch: Ziegelind. Dtsch (junio 1963), 16, 11, 387-390.

(35) Regard critique sur la fabrication des agrégats légers. Brick Clay Rec. (noviembre 1963), 143, 5, 54-56.

(36) Béton "Céramsite", Ceram. (octubre 1963), 14, 176, 58.

(37) J. E. Conley, H. Wilson y T. A. Klinefelter: British Clay Worker, octubre 1949 a septiembre 1950.

(38) K. MEYER: Tonindustrie Zeitung Dtsch. enero 1952.

(39) Anónimo: Factors in making lightweight aggregate by sintering process. Brick and Clay Record (junio 1950). 